\title{
FONCTIONS TOTALEMENT CROISSANTES: UNE CORRECTION
}

\author{
JEAN-PIERRE GRENIER
}

(Communicated by Andrew M. Bruckner)

\begin{abstract}
Resume. On montre, à l'aide d'un contre exemple qu'un des énoncés classique de la théorie des fonctions totalement croissantes (A. Revuz) est faux, puis on en donne une version corrigée.
\end{abstract}

ABSTRACT. Using a counterexample we show that a classical result about the totally increasing functions (A. Revuz) is false, then we give a right version of it.

Soit $(E, \leq)$ un demi treillis inférieur (i.e., toute paire $\{x, y\}$ d'éléments de $E$ a une borne inférieure notée $x \wedge y$ ) muni d'une topologie $T$. Lorsque $A$ est une partie de $E, A^{c}$ est le complémentaire de $A$. Pour tout $t \in E$, on définit le cône négatif $C_{-}(t)=\{x \in E \mid x \leq t\}$ et le cône positif $C_{+}(t)=$ $\{x \in E \mid x \geq t\}$; plus généralement pour toute partie finie $P$ de $E$ on pose $C_{-}(x ; P)=C_{-}(x) \backslash\left(\bigcup\left\{C_{-}(p) \mid p \in P\right\}\right)$. On sait que l'anneau $\mathscr{A}$ engendré par l'ensemble $\mathscr{G}=\left\{C_{-}(x) \mid x \in E\right\}$ est l'ensemble des réunions finies disjointes de parties $C_{-}(x ; P)$ (voir [1]). Pour tout $t \in E$, la trace sur $C_{+}(t)$ du filtre des voisinages de $t$ engendre le filtre des voisinages à droite de $T$.

On considère les axiomes suivants introduits par A. Revuz [1]: On dit que $(E, \leq, T)$ satisfait l'axiome

Xa: lorsque pour tout $x \in E, C_{-}(x)$ est compact.

$\mathrm{X} \alpha$ : lorsque pour tous $x, y \in E$, l'intervalle $[x, y]=C_{+}(x) \cap C_{-}(y)$ est compact.

$\mathrm{X} \alpha^{\prime}$ : lorsque pour tout $x \in E$, il existe $y \in E$ tel que $x$ est intérieur à $C_{+}(y)$.

$\mathrm{Xb}$ : lorsque l'application $E \times E \rightarrow E$

$$
(x, y) \mapsto x \wedge y \quad \text { est continue à droite. }
$$

Xc: lorsque pour tout $x \in E$ non maximal, et tout voisinage à droite $V$ de $x$, il existe $y \in V$ tel que $C_{-}(x)$ est contenu dans l'intérieur de $C_{-}(y)$.

$\mathrm{Xc}^{\prime}$ : lorsque pour tout $a \in E$, l'ensemble $C_{-}(a)$ muni de la topologie induite satisfait Xc.

On rappelle les deux résultats suivants [1, pp. 187-210].

Received by the editors January 8, 1994.

1991 Mathematics Subject Classification. Primary 28C99; Secondary 06B30. 
Théorème 1. (a) Soit une application $f: E \rightarrow \mathbb{R}$. Il existe une unique mesure $\nu$ finiment additive définie sur l'anneau $\mathscr{A}$ telle que pour tout $x \in E$, on ait $\nu\left(C_{-}(x)\right)=f(x)$. La fonction $f$ est totalement croissante lorsque $\nu$ est à valeurs dans $\mathbb{R}+$.

(b) Si $(E, \leq, T)$ satisfait $\left(\mathrm{Xa}, \mathrm{Xb}, \mathrm{Xc}^{\prime}\right)$ et si l'application $f$ est continue à droite sur $E$ et totalement croissante, alors $\nu$ est dénombrablement additive, ce qui assure par le théorème de Carathéodory qu'elle se prolonge d'une manière unique en une mesure $\sigma$-additive définie sur le $\sigma$-anneau $\sigma(\mathscr{A})[2, p$. 208].

Le théorème 2, p. 237 de [1], affirme:

"Théorème 2." Soit $(E, \leq, T)$ un demi treillis topologique sans élément maximal qui satisfait les axiomes $\left(\mathrm{X} \alpha, \mathrm{X} \alpha^{\prime}, \mathrm{Xb}, \mathrm{Xc}\right)$; on suppose que pour tout $x \in E$ le complémentaire $\left(C_{+}(x)\right)^{c}$ est non vide. Si $f: E \rightarrow \mathbb{R}$ est totalement croissante et continue à droite et a pour limite 0 suivant le filtre engendré par l'ensemble $\left\{\left(C_{+}(x)\right\}^{c} \mid x \in E\right\}$, alors il existe une mesure $\nu \sigma$-additive sur $E$ telle que, pour tout $x \in E, \nu\left(C_{-}(x)\right)=f(x)$.

Cet énoncé est faux. L'erreur dans la démonstration de A. Revuz (p. 236) est due au fait que le "complété" $\tilde{X}$ de $X$ ne satisfait pas Xc. L'énoncé reste faux si $E$ a des points maximaux (on construit un contre-exemple en supprimant les points $b_{-n}$ dans le contre-exemple suivant.

Contre-exemple. On construit l'ensemble $(E, \leq)$ par récurrence. On part d'un individu $b_{0}$ qui constitue la génération $n^{\circ} 0$. On munit $b_{0}$ d'ancêtres $b_{-1}$, $b_{-2}, \ldots$ qui forment les générations $-n$ où $n \in \mathbb{N}^{*}$. On munit $b_{0}$ de trois fils:

son fils gauche $a_{0}$,

son fils droit $a_{1}$,

son fils universel $b_{1}$.

Ces trois descendants constituent la $1^{\text {ère }}$ génération (voir Figure 1).

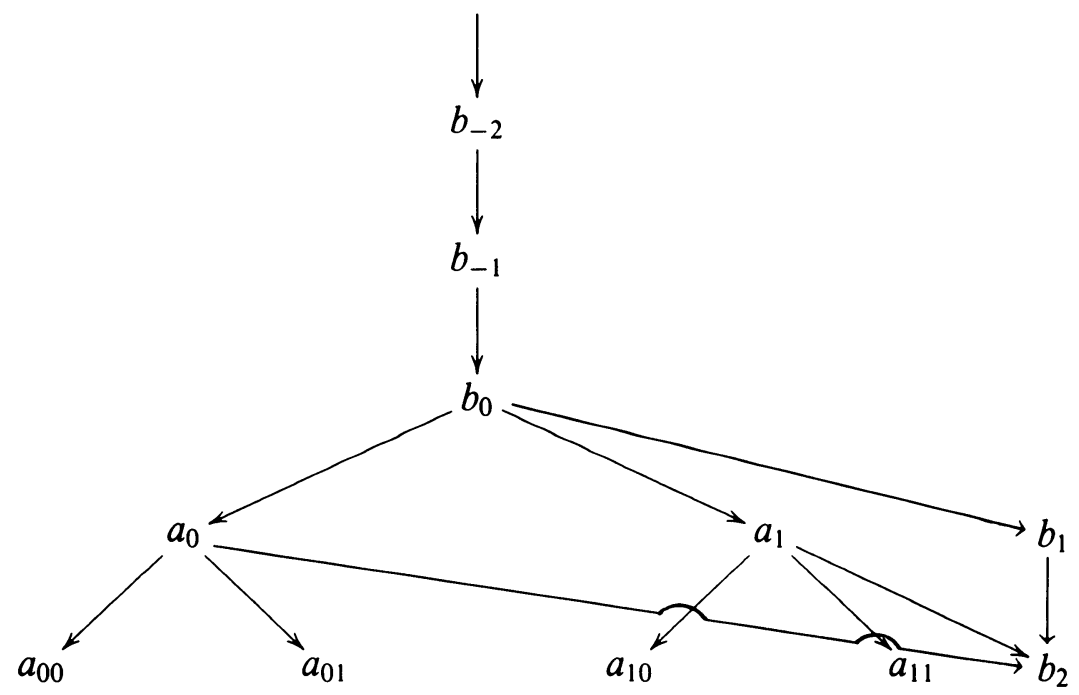

FIGURE 1 
Supposons que pour $n \geq 1$, la $n^{\text {ième }}$ génération est constituée des $2^{n}$ éléments $a_{s}$ où $s \in\{0,1\}^{n}$ et de $b_{n}$. On pose $G_{n}=\left\{b_{n}\right\} \cup\left\{a_{s} \mid s \in\{0,1\}^{n}\right\}$. Les trois fils de $a_{s}$ sont:

son fils gauche $a_{s 0}$,

son fils droit $a_{s 1}$,

son fils universel $b_{n+1}$.

L'unique fils de $b_{n}$ est $b_{n+1}$.

$\mathrm{La}(n+1)^{\text {ième }}$ génération est ainsi définie par récurrence.

On définit l'ordre sur $E$ par la relation:

$$
x \leq y \Leftrightarrow x \text { est un descendant de } y \text { ou } x=y .
$$

$(E, \leq)$ est un demi-treillis; en effet:

Si $x \leq y$ alors $x \wedge y=x$.

Si $x$ et $y$ ne sont pas comparables, soit $n$ la génération de $x$ et $m$ celle de $y$, alors $x \wedge y=b_{\text {sup }(n, m)+1}$.

On munit $E$ de la topologie discrète. Les axiomes $\left(\mathrm{X} \alpha, \mathrm{X} \alpha^{\prime}, \mathrm{Xb}, \mathrm{Xc}\right)$ sont vérifiés de manière évidente.

On considère l'application $f$ suivante: Pour tout entier $n \in \mathbb{N}$, on pose $f\left(b_{-n}\right)=1$ et pour tout entier $\geq 1$, si $x$ fait partie de la $n^{\text {ième }}$ génération, on pose

$$
f(x)= \begin{cases}1 / 2^{n} & \text { si } x=a_{s}, \\ 0 & \text { si } x=b_{n}\end{cases}
$$

Il est clair de $f$ satisfait les hypothèses du "théorème 2". Pour tout $x \in E$, on a $\nu(\{x\})=0$, de sorte que $f$ est bien totalement croissante. Puisque $G_{n}$ est un ensemble fini, on a $\nu\left(G_{n}\right)=0$, donc $\sum_{n=0}^{+\infty} \nu\left(G_{n}\right)=0$. D'autre part $C_{-}\left(b_{0}\right)=\bigcup_{n=0}^{+\infty} G_{n}$, et donc, $\nu\left(\bigcup_{n=0}^{+\infty} G_{n}\right)=f\left(b_{0}\right)=1$. Ainsi, $\nu$ ne saurait être dénombrablement additive.

Nous allons maintenant établir un énoncé exact destiné à rectifier l'énoncé précédent.

Théorème 3. Supposons que $(E, \leq, T)$ satisfait les axiomes $\left(\mathrm{X} \alpha, \mathrm{Xb}, \mathrm{Xc}^{\prime}\right)$ et la propriété (1) suivante:

(1) Pour tout $a \in E$, pour tout $\varepsilon>0$, il existe $b \in E$ et une partie $S \in \mathscr{A}$ tel que $C_{-}(a) \backslash[b, a] \subset S$ et $\nu(S)<\varepsilon$.

Alors $\nu$ est dénombrablement additive.

Démonstration. On écrira $\bigcup_{k}$ et $\sum_{k}$ au lieu de $\bigcup_{k \in \mathbb{N}}$ et $\sum_{k \in \mathbb{N}}$. Donnons nous une partie $C=C_{-}(a ; P) \in \mathscr{A}$ et une suite de parties $C_{k}=C_{-}\left(x_{k} ; P_{k}\right)$ deux à deux disjointes de $\mathscr{A}$ telle que $C=\bigcup_{k} C_{k}$. On sait que l'on peut supposer $P \subset C_{-}(a)$ et pour tout $k \in \mathbb{N}, C_{-}\left(x_{k} ; P_{k}\right) \subset C_{-}(a)$; donnons nous $\varepsilon>0, b$ et $S$ comme dans l'énoncé choisissons de plus $b \leq \wedge P$. Il suffit de montrer que $\nu(C) \leq \sum_{k} \nu\left(C_{k}\right)$.

L'ensemble $[b, a]$ est compact, on applique le théorème 1: Il existe une mesure $\sigma$-additive $\mu$ sur $[b, a]$ telle que pour tout $x \in[b, a]$ on a $\mu([b, x])=$ $f(x)=\nu\left(C_{-}(x)\right)$. On a $\nu(C)=\mu(C \cap[b, a])$. On pose $C^{\prime}=C \backslash S$, on voit que $C^{\prime} \in \mathscr{A}, C^{\prime} \subset[b, a]$ et $\nu(C) \leq \nu\left(C^{\prime}\right)+\varepsilon$. D'autre part, $C^{\prime}=\bigcup_{k}\left(C_{k} \backslash S\right)$ (union disjointe) d'où $\mu\left(C^{\prime}\right)=\sum_{k} \mu\left(C_{k} \backslash S\right)$. Enfin, comme $\mu\left(C_{k} \backslash S\right)=\nu\left(C_{k} \backslash S\right)$ $\leq \nu\left(C_{k}\right)$, on obtient $\mu\left(C^{\prime}\right) \leq \sum_{k} \nu\left(C_{k}\right)$, d'où $\nu(C) \leq \sum \nu\left(C_{k}\right)+\varepsilon$, ceci pour tout $\varepsilon>0$ ce qui démontre le résultat. 
Remarques. Le théorème 3 reste vrai en remplacant dans la propriété (1) l'hypothèse ' $S \in \mathscr{A}$ ' par' $\mathscr{S}$ est réunion dénombrable d'éléments de $\mathscr{A}$ ', mais la démonstration est bien plus longue.

\section{BIBLIOGRAPHIE}

1. A. Revuz, Fonctions croissantes et mesures sur les espaces topologiques ordonnés, Ann. Inst. Fourier $6(1955,1956), 187,269$.

276, Rue des Glycines, 45160 Olivet, France 